\title{
Segmental Conduction Block in a Low-Voltage Area Suppressed Macro-Reentrant Ventricular Tachycardia after Surgical Repair of Tetralogy of Fallot
}

\author{
Masaomi Chinushi ${ }^{1}$, Satoru Komura ${ }^{2}$, Hiroshi Furushima ${ }^{2}$ and Yoshifusa Aizawa ${ }^{2}$
}

\begin{abstract}
Macro-reentrant ventricular tachycardia (VT) developed in a 20-year-old man, 17 years after surgical repair of tetralogy of Fallot. Activation mapping of the VT revealed its counterclockwise propagation around the right ventricle, and through a critical pathway between a transannular patch and the tricuspid annulus. This critical pathway was $6 \mathrm{~cm}$ long and contained myocardium with a normal amplitude, while the area of low voltage was limited adjacent to the transannular patch. A linear lesion was created by radiofrequency energy delivered only to the low voltage area. After ablation, the activation wavefront through the low voltage area was blocked, and VT became non-inducible.
\end{abstract}

Key words: macro-reentrant ventricular tachycardia, tetralogy of Fallot, radiofrequency ablation, activation mapping

(Inter Med 48: 1021-1023, 2009)

(DOI: 10.2169/internalmedicine.48.2048)

\section{Introduction}

Ventricular tachycardia (VT) may develop and cause sudden death late after repair of tetralogy of Fallot (1). This usually reentrant VT is supported by a circuit around an anatomical or surgical right ventricular (RV) barrier (2-6). It can be treated by radiofrequency (RF) catheter ablation, to create a line of complete conduction block across the isthmus borrowed by the activation wavefront (2-6). This, however, may be challenging or unattainable if the isthmus is inordinately long, or if it is located within hypertrophied myocardium. We have recently created a line of segmental block within a low-voltage area, which eliminated a macroreentrant VT.

\section{Case Report}

A 20-year-old man who had undergone surgical repair of tetralogy of Fallot at age 3 was admitted for recurrent syncope. The operation consisted of closure of the ventricular septal defect with a patch, and plasty of the RV outflow tract, using a transannular patch. The 12-lead surface electrocardiogram at rest showed normal sinus rhythm, 1st degree atrioventricular block and complete right bundle branch block. QRS complex during sinus rhythm was $180 \mathrm{~ms}$. An echocardiogram revealed the presence of RV hypertrophy and enlargement. The left ventricular ejection fraction was $72 \%$. Brain computed tomography and electroencephalogram were normal.

After informed consent was obtained, a cardiac catheterization and electrophysiologic study were performed. Measurements of the intracardiac pressures showed a small RV $(46 / 6 \mathrm{mmHg}) \rightarrow$ main pulmonary artery $(37 / 6 \mathrm{mmHg})$ gradient. The left ventriculogram and coronary angiograms were normal.

At electrophysiologic study, during sinus rhythm, the $\mathrm{AH}$ and $\mathrm{HV}$ intervals were $300 \mathrm{~ms}$ and $45 \mathrm{~ms}$, respectively. Sinus node function was normal and the Wenckebach cycle length during atrial pacing was $500 \mathrm{~ms}$. Electro-anatomical mapping with a CARTO Navigation System (BiosenseWebster, Diamond Bar, CA) showed the presence of a scar

${ }^{1}$ School of Health Science, Niigata University School of Medicine, Niigata and ${ }^{2}$ The First Department of Internal Medicine, Niigata University School of Medicine, Niigata

Received for publication January 12, 2009; Accepted for publication March 16, 2009

Correspondence to Dr. Masaomi Chinushi, masaomi@clg.niigata-u.ac.jp 

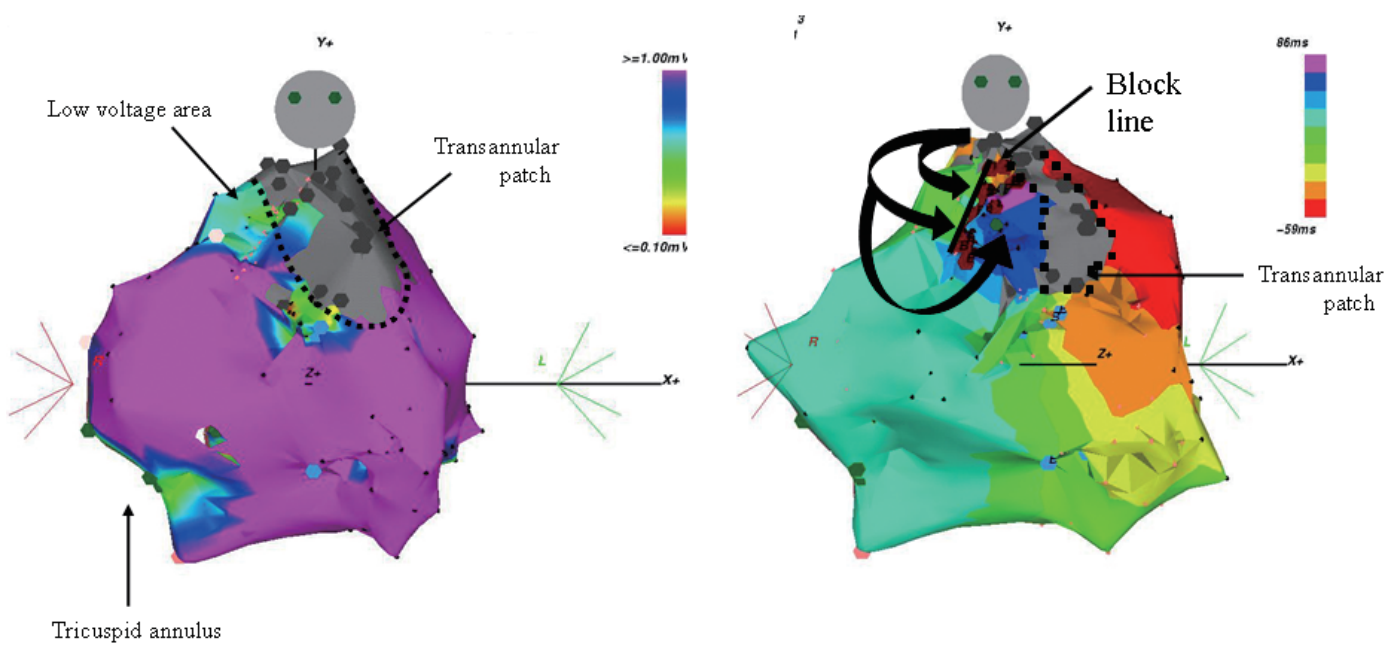

Figure 1. Voltage mapping and activation mapping. During sinus rhythm (panel A), an area where the endocardial electrograms were $<1.0 \mathrm{mV}$ in amplitude, limited beside the transannular patch. A dense scar is present in the region of the transannular patch and it is marked by black dots. After segmental liner ablation (panel B), during RV septal pacing, the activation wavefront was blocked in that area. The ablation points are marked by brown tags.

$(<0.1 \mathrm{mV})$ in the region of the transannular patch, and endocardial electrograms $<1.0 \mathrm{mV}$ in amplitude were recorded in the area beside the transannular patch during the sinus rhythm (Fig. 1A). In contrast, $>3.0 \mathrm{mV}$ endocardial signals were present in most of the other RV myocardial regions. Double RV extrastimuli reproducibly induced sustained monomorphic VT with a left bundle branch block pattern. The cycle length of the VT was 300-310 ms. Activation wavefront of the VT was propagating counterclockwise around the RV and the propagation time $(278 \mathrm{~ms})$ almost covered the VT cycle length (Fig. 2). Concealed entrainment was documented during rapid pacing, through a $6 \mathrm{~cm}$ long pathway situated between the transannular patch and the tricuspid annulus. Mid-diastolic potential was also recorded at the site (Fig. 2). During the VT, the activation wavefront propagated across the low voltage area adjacent to the transannular patch (Fig. 2), but entrainment study at the site could not be performed because pacing at the maximum output $(10 \mathrm{~V} \times 2 \mathrm{~ms})$ could not capture the local electrograms. Since the creation of continuous block line across the entire length of the reentrant pathway seemed problematic, RF energy was applied to the area of low voltage, with a view to create a line of conduction block in that region, which we subsequently confirmed by activation mapping (Fig. 1B). After the procedure, VT became non-inducible. The patient accepted treatment of cardioverter defibrillator and was discharged from the hospital after the implantation. He has remained free of VT recurrence over a 24-month period.

\section{Discussion}

Previous studies have shown that macro-reentrant VT af- ter surgical repair of tetralogy of Fallot may be supported by an isthmus of slow conduction between the RV incision scar and the pulmonic valve, or between surgical transannular, or ventricular septal defect patches and the tricuspid annulus (2-6). A linear RF ablation which blocked conduction throughout the isthmus effectively eliminated VT in some reports (2-6). In the present case, activation mapping and concealed entrainment indicated that the critical area supporting the reentrant mechanism was located between the transannular patch and the tricuspid annulus. Zeppenfeld et al recently reported that critical isthmus of macro reentrant VT after surgical repair of tetralogy of Fallot was most frequently located in this portion, and such critical isthmus could be identified using 3D voltage mapping during sinus rhythm (6). However, in our patient, this area was $6 \mathrm{~cm}$ long, and most of the myocardium it contained was associated with electrograms that were normal in voltage. Since we also observed RV hypertrophy on the echocardiogram, the creation of complete linear block across that area appeared difficult without using an irrigation or cooled tip ablation system, thus far unavailable in Japan. Zeppenfeld et al also described similar difficulties in their report (6).

The zone of slow conduction associated with the induction of VT was most likely located in the area of low voltage, which we targeted when creating the linear RF lesion. We think that RF ablation prevented the completion of the initial process of the reentrant VT (slow conduction and/or unidirectional block). Although the reentrant pathway of the VT remained in the RV, we think that the conduction velocity in the normal myocardium was not slow enough to produce a stable reentry in the RV. To the best of our knowledge, this is the first report of segmental linear conduction block in a low voltage zone, which we confirmed during RV 


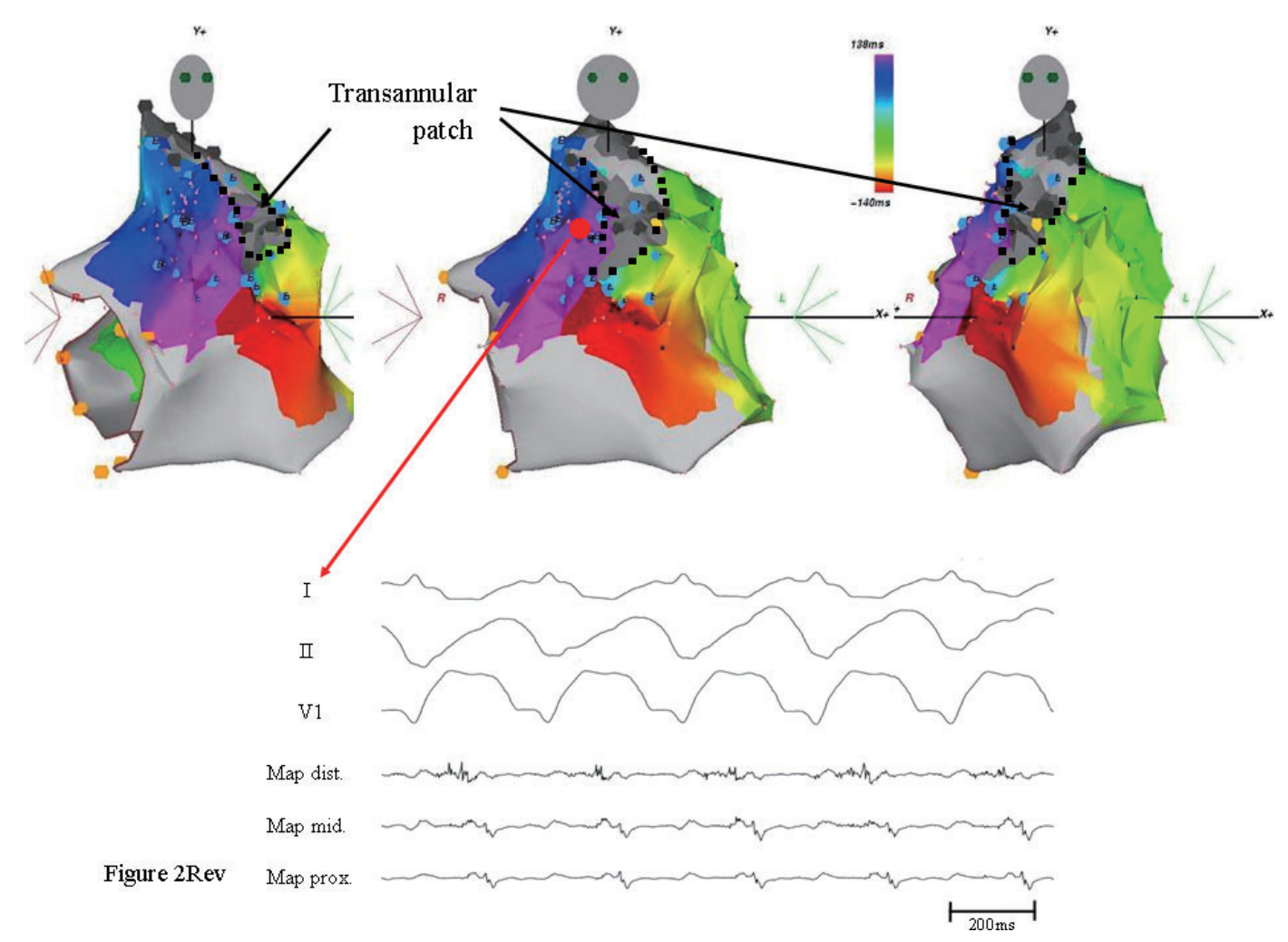

Figure 2. Activation mapping using a quadripolar catheter. The VT activation wavefront circulates counterclockwise around the RV (upper panels) and almost covered the VT cycle length. The critical area was identified between the edge of the transannular patch and the tricuspid annulus, where a mid-diastolic potential was recorded (lower panel). Activation of the VT propagated through the low voltage area of the RV which was shown in Figure 1A. The location of the transannular patch is marked by black dots. Map dist.=distal pair of the mapping catheter, Map mid.=bipolar electrogram recorded from the second and third electrodes of the mapping catheter, Map prox.=proximal pair of the mapping catheter.

pacing, followed by non-inducibility and long-term elimina- tion of a macro-reentrant VT.

\section{References}

1. Gatzoulis MA, Balaji S, Webber SA, et al. Risk factors for arrhythmia and sudden cardiac death late after repair of tetralogy of Fallot: a multicentre study. Lancet 356: 975-981, 2000.

2. Gonska BD, Cao K, Raab J, Eigster G, Kreuzer H. Radiofrequency catheter ablation of right ventricular tachycardia late after repair of congenital heart defects. Circulation 94: 1902-1908, 1996.

3. Furushima H, Chinushi M, Sugiura H, et al. Ventricular tachycardia late after repair of congenital heart disease: efficacy of combination therapy with radiofrequency catheter ablation and class III antiarrhythmic agents and long-term outcome. J Electrocardiol 39: 219-224, 2006.

4. Stevenson WG, Delacretaz E, Friedman PL, Ellison KE. Identifi- cation and ablation of macroreentrant ventricular tachycardia with the CARTO electroanatomical mapping system. Pacing Clin Electrophysiol 21: 1448-1456, 1998.

5. Chinushi M, Aizawa Y, Kitazawa H, Kusano Y, Washizuka T, Shibata A. Successful radiofrequency catheter ablation for macroreentrant ventricular tachycardias in a patient with tetralogy of Fallot after corrective surgery. Pacing Clin Electrophysiol 18: 1713-1716, 1995.

6. Zeppenfeld K, Schalij MJ, Bartelings MM, et al. Catheter ablation of ventricular tachycardia after repair of congenital heart disease. Electroanatomic identification of the critical right ventricular isthmus. Circulation 116: 2241-2252, 2007.

(C) 2009 The Japanese Society of Internal Medicine http://www.naika.or.jp/imindex.html 